\title{
33
}

\section{Managing Virtual Paths on Xunet III: Architecture, Experimental Platform and Performance}

\author{
Nikos G. Aneroussis and Aurel A. Lazar \\ Department of Electrical Engineering and \\ Center for Telecommunications Research \\ Rm. 801 Schapiro Research Bldg. \\ Columbia University, New York, NY 10027-6699 \\ e-mail: \{nikos,aurel\}@ctr.columbia.edu \\ Tel: (212) 854-2399
}

\begin{abstract}
An architecture for integrating the Virtual Path service into the network management system of future broadband networks is presented. Complete definitions and behavioral descriptions of Managed Object Classes are given. An experimental platform on top of the XUNET III ATM network provides the proof of concept. The Xunet manager is equipped with the necessary monitoring tools for evaluating the performance of the network and controls for changing the parameters of the VP connection services. Performance data from Xunet is presented to highlight the issues underlying the fundamentals of the operation of the VP management model such as the trade-off between throughput and call processing load.
\end{abstract}

\section{Keywords}

ATM, Quality of Service, Virtual Path Management, Performance Management, Gigabit Testbeds, Xunet

\section{INTRODUCTION}

Central to the operation of large scale ATM networks is the configuration of the Virtual Path (VP) connection services. VPs in ATM networks provide substantial speedup during the connection establishment phase at the expense of bandwidth loss due to reservation of network resources. Thus, VPs can be used to tune the fundamental trade-off between the cell throughput and the call performance of the signalling system. They can also be used to provide dedicated connection services to large customers such as Virtual Private Networks (VPNs). This important role of VPs brings forward the need for a comprehensive management architecture that allows the configuration of VP connection services and the evaluation of the resulting network performance. Furthermore, call-level performance management is essential to the operation of large ATM networks for routing decisions and for long term capacity planning.

The review of the management efforts for ATM broadband networks reveals that there has been little work regarding the management of network services. In [OHT93], an OSI-based management system for testing ATM Virtual Paths is presented. The system is used exclusively for testing the cell-level performance of Virtual Paths, and allows the control of cell generators and the retrieval of information from monitoring sensors. The system is designed for testing purposes only and does not have the capability to install Virtual Paths, regulate their networking capacity, or measure any call-level statistics.

A more complete effort for standardizing the Management Information Base for ATM LANs that meets the ATM Forum specifications is currently under way in the Internet Engineering Task Force (IETF) [IET94]. This effort focuses on a complete MIB specification based on the SNMP standard for configuration management, including VP configuration management. Performance management is also considered but at the cell level only. 
The ICM RACE project [ICM93] is defining measures of performance for ATM networks both at the call and at the cell level and the requirements for Virtual Path connection management. It is expected to deliver a set of definitions of managed objects for VP management and demonstrate an implementation of the architecture.

In [ANE93] we have described a network management system for managing (mostly monitoring) low level information on XUNET III. Our focus in this paper is on managing services, in particular, services provided by the connection management architecture. In order to do so, there is a need to develop an understanding of the architecture that provides these services. The integration of the service and network management architectures can highly benefit from an overall network architecture model [LAZ93].

Within the context of a reference model for network architectures that we have previously published [LAZ92], we present an architectural model for VP connection setup under quality of service constraints. The architecture is integrated with the OSI management model. Integration here means that VPs set up by the connection management system can be instrumented for performance management purposes. The reader will quickly recognize that this instrumentation is representative for a large class of management problems such as billing (accounting), configuration management, etc.

We emphasized the following capabilities: monitoring Virtual Circuits (VCs) independently; monitoring and control of Virtual Paths; monitoring the call-level performance by computing statistics such as call arrival rates, call blocking rates, call setup times, etc.; control of the call-level performance through allocation of network resources to Virtual Paths, and control of other operating parameters of the signalling system that influence the call-level performance, such as retransmission time-outs, call setup time-outs, call-level flow control, etc.

We have tested our overall management system on the Xunet ATM broadband network that covers the continental US. Finally, we have taken measurements that reveal the fundamental trade-off between the throughput and the signalling processing load as well as other quantities of interest that characterize the behavior of broadband networks.

This paper is organized as follows. Section 2 presents the architectural framework for managing VP connection services. Section 3 describes the Xunet III experimental platform and the implementation details of the VP management system. Network experiments with the objective of evaluating the management model and the performance of the network under several configurations of the VP connection services are presented in Section 4. Finally, Section 5 summarizes our findings and presents the directions of our future work.

\section{ARCHITECTURE}

In this section we present an overall architectural framework for managing the performance of VP services on broadband networks. Underlying our modeling framework is the Integrated Reference Model (IRM) described in Section 2.1. The VP architecture embedded within the IRM is discussed in Section 2.2. The management architecture is outlined in section 2.3. Finally, in section 2.4 the integration of the service and network management architectures is presented.

\subsection{The Integrated Reference Model}

To overcome the complexity problems in emerging broadband networks - caused by the variety of communication services to be provided, the required quality of service guarantees, the large number of network nodes, etc. - there is an urgent need for integrating network management, service management and real-time control tasks into a consistent framework. To this end, we have developed an overall model for network architectures called the Integrated Reference Model (IRM) [LAZ92]. In this model, the key role for network integration is played by the network telebase, a distributed data repository that is shared among network mechanisms. 
The IRM incorporates monitoring and real-time control, management, communication, and abstraction primitives that are organized into five planes: the network management or $\mathrm{N}$-plane, the resource control or M-plane, the data abstraction and management or D-plane, the connection management or C-plane and the user information transport or U-plane (Figure 1). The subdivision of the IRM into the N-, M- and C-

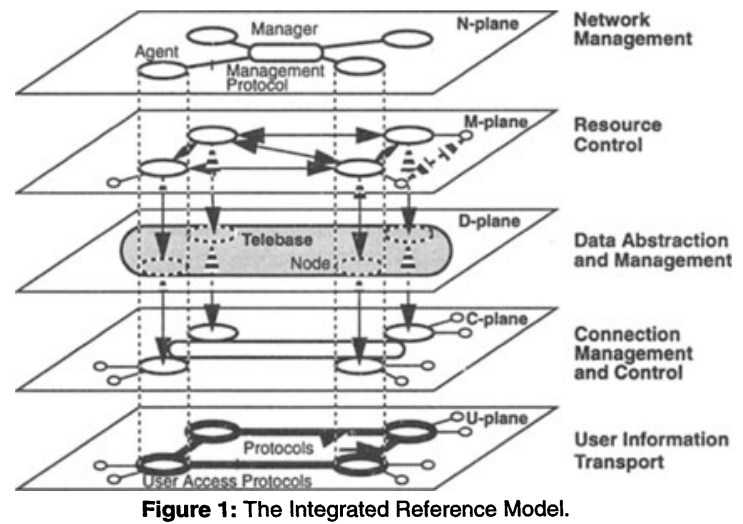

plane on the one hand, and the U-plane on the other, is based on the principle of separation between controls and communications. The separation between the $\mathrm{N}$ - and, $\mathrm{M}$ - and $\mathrm{C}$-planes is primarily due to the different time-scales on which these planes operate.

The $\mathrm{N}$ - plane covers the functional areas of network management, namely, configuration, performance, fault, accounting and security management. Manager and agents, its basic functional components, interact with each other according to the client-server paradigm. The M-plane consists of the resource control and the C-plane of connection management and control. The M-plane comprises the entities and mechanisms responsible for resource control, such as cell scheduling, call admission, and call routing; the C-plane those for connection management and control. The user transport or U-plane models the protocols and entities for the transport of user information. Finally, the data abstraction and management or D-plane (the Telebase) implements the principles of data sharing for network monitoring, control and communication primitives, the functional building blocks of the N-, M-, and $\mathrm{C}$ - and $\mathrm{U}$-plane mechanisms. (A mechanism is a functional atomic unit that performs a specific task, such as setting up a virtual circuit in the network [LAZ93]).

\subsection{VP Architecture}

The VP architecture closely follows the organization proposed by the IRM. It can be divided in two parts: the first part describes a model for establishing VPs, and the second presents a model for VP operation during the call setup procedure. In either case, central to the VP architecture is the D-plane of the IRM. The D-plane contains all information regarding the configuration and operational state of VPs and is used by the algorithms of the other planes both for monitoring and control operations.

The establishment of VPs is performed by the signalling system. The latter resides in the C-plane. A signalling protocol is used to establish a VP hop by hop. At every node along the route of the VP, the necessary networking capacity must be secured from the output link that the VP is traversing. The networking capacity of links is described by the Schedulable Region (SR) [HYM91], and of VPs by the Contract Region (CR) [HYM93b]. Informally, the Schedulable Region is a surface in a $k$ dimensional space (where $k$ is the number of traffic classes), that describes the allowable combinations of calls from each traffic class that can be accepted on the link and be guaranteed Quality of Service. The Contract Region is a region of the SR reserved for exclusive use by the VP. If the requested capacity allocation of a VP cannot be 
achieved, the allocated capacity at the end of the VP establishment phase is the minimum capacity available on the corresponding links (best effort resource allocation). The set of all VPs in the network, characterized by their route, Contract Region and associated configuration information, comprise the $V P$ distribution policy. The VP distribution policy is stored in the D-plane.

An admission control algorithm located in the M-plane formulates the admission control policy (ACP), which is encoded as an object in the D-plane. The ACP is used by the signalling algorithm of the C-plane to make admission control decisions for incoming call requests. Thus, the VP architecture represents a connection service installed in the D-plane.

Figure 2 shows the interaction between entities in the various planes of the IRM that provide the VP con-

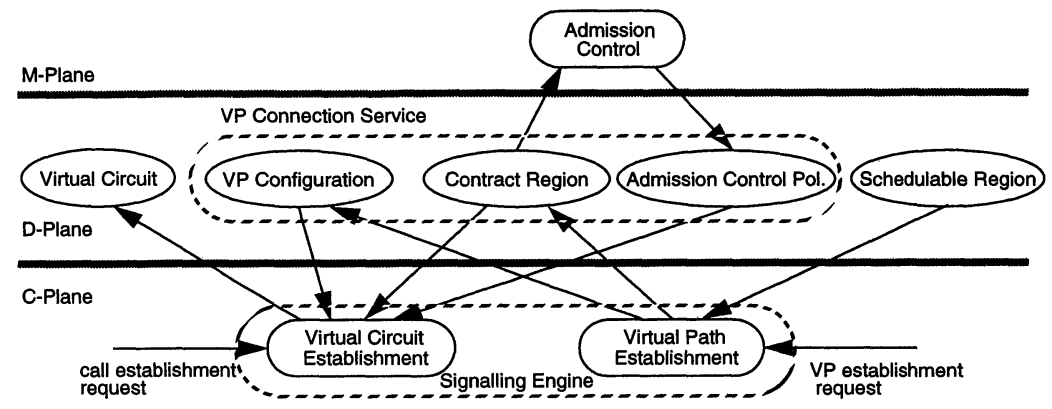

Figure 2: Flow of Information during Installation and Operation of the VP Connection Service.

nection service. During the VP establishment phase, the signalling engine creates a set of 3 objects in the D-plane: the CR, ACP and VP Configuration objects. The VP configuration object contains general VP configuration information such as the input and output port numbers, the allocation of the VCI space, the VP operational state, etc.

During the VC establishment phase, the signalling engine reads the VP configuration object to determine if the VP can be used to reach the desired destination. It also reads the CR and ACP objects to examine if the call can be admitted on the VP. When the call has been established, a Virtual Circuit object is created in the D-plane that contains all necessary information for the VC. This information includes the VP Identifier (VPI) and VCIdentifier (VCI), the traffic descriptor used to allocate resources, and other parameters for performance monitoring.

VPs can be used in two ways. If the VP is terminated at the Customer Premises Equipment (CPE), the customer is controlling the VP admission controller. In this case the VP can be regarded as a dedicated virtual link (or pipe) of a rated networking capacity. A network composed of such VPs terminated at the customer premises is also known as a Virtual Private Network (VPN). The Network Manager has the capability to configure and maintain a VPN by managing the individual VP components according to the customer requirements.

Alternatively, the termination of VPs may not be visible to the network customer. In this case, VPs are used by the network operator to improve the performance of the signalling system, the availability of resources between a pair of nodes, or even improve certain call level measures of Quality of Service for the customer, such as call setup time and blocking probability.

\subsection{Management Architecture}

The Management Architecture builds on the OSI Management model. According to this model, network entities (either physical, like hardware modules, or logical, like virtual circuits) are mapped into "Managed Objects" for monitoring and control purposes. The managed objects are also referred to as logical 
objects and the network entities that they represent as real objects. A Management Agent contains the information about the managed objects in the Management Information Base (MIB). The MIB is an objectoriented database. Managed objects are characterized by a set of attributes that reflect the state of the corresponding real object and behavioral information, which defines the result of management operations on the managed object. A proprietary protocol can be used for linking the state of every real object to its logical counterpart in the MIB. The Manager connects to the agent(s) and performs operations on the objects in the MIB using CMIP (the Common Management Information Protocol). These operations are of synchronous nature, i.e., they are initiated by the manager who, then, waits for a reply from the agent(s). Events of asynchronous nature (notifications) such as hardware faults can be emitted from the agent(s) to the manager using the event reporting primitive of CMIP.

Management operations take place in the N-plane of the IRM(Figure 1). The MIB of every agent is located in the D-plane of the IRM. As a result, the linking of the logical objects in the MIB with real objects is done within the D-plane [TSU92]. Control operations from the manager applied to objects in the MIB are reflected in the state of the real objects of the D-plane, which in turn, affect the behavior of the algorithms in the C- and M-planes. Conversely, the change of state of the real objects in the D-plane will cause an update of the state of the logical objects in the MIB.

Therefore, in our model, monitoring and control of the VP architecture is possible by defining the appropriate managed objects in the MIB and linking them to the appropriate entities of the D-plane. What managed objects to define, how to integrate them in the D-plane and how to define their behavior will be the topic of the following section.

\subsection{Integration of the VP and Management Architecture}

The purpose of this section is to describe the object level model for VP management and its integration within the D-plane. Management of VPs takes place in the N-plane. The network manager decides on a VP distribution policy and implements this policy by issuing commands to the agents installed across the network. Although the capabilities to install and control VPs are essential requirements to implement a VP distribution policy, it is also essential for the manager to evaluate the performance of the network under a given VP distribution policy. For this reason, a generic performance management model (in addition to the VP management model) both at the call and the cell level becomes necessary. Note, however, that VP management operations stem from the call-level performance management model, and therefore, the VP management model can be considered as part of the latter.

The performance management model consists of a set of quantities that reflect network performance, and a set of controls that affect this performance. A set of rules use the performance measures to derive the necessary controls that will reach a performance objective. At the call level, the quantities of interest are the call arrival rate, the call blocking rate, the call setup time, the signalling load, etc.; at the cell level, the cell arrival rate, cell throughput and end-to-end delay. These quantities together with a set of controls must appear in the definition of Managed Object Classes (MOCs) for performance management.

In our model, one agent is installed at every ATM switch. The agent processes the information on call attempts from every input port. For each successful call attempt, an object of class VcEntity is created for the corresponding Virtual Circuit connection (VC). Each VC object contains configuration information such as the number of the input and output slot, Virtual Path Identifier(VPI) and Virtual Circuit Identifier (VCI). In ATM terminology, this implies that the VC object models the input and output Virtual Circuit Link (VCL) at every switch. Thus, the end-to-end management of a VC that spans many switches (and hence has one instance in each OSI agent at every switch) is achieved by managing the individual objects in combination. Additional attributes for each VC include the call establishment time, traffic descriptor (composed of a service class characterization and the allocated networking capacity in kilobits per second), adaptation layer information and source/destination end-user service information. The package cellPerformancePackage contains attributes associated with the cell-level performance related parameters, and will be described below. 
The class VirtualPath derived from Top is used to describe a VP. The VP object in analogy with the VC object is comprised of an incoming and an outgoing part at every switch. At the VP source or termination point, the VP has only an outgoing / incoming part respectively. Attributes used to describe the configuration of the Virtual Path are: vpIdentifier (VPI), vpSource, vpDestination (VP source and termination address), circuitCapacity and timeEstablished. The VP object at the source also contains a callPerformancePackage, and an admissionControllerPackage. These will be described below.

The class Link is derived from Top and is used to model input or output network links. The mandatory attributes for this class are linkType (input or output), linkModuleDescription (describes the hardware of the link interface), linkSource, linkDestination and linkSlotNumber (the slot number the link is attached to). If it is an output link, it contains a callPerformancePackage, and an admissionControllerPackage.

The class SourceDestination is used to describe the call level activity between a pair of nodes, and can be used to evaluate the call level performance in an end-to-end fashion. A Source-Destination (SD) object exists in the agent if there is call-level activity between the two nodes, and the source node is either the local switch, or a directly attached User-Network Interface (UNI). The SD object contains the following attributes: sourceNodeAddress and destinationNodeAddress and a callPerformancePackage.

The callPerformancePackage is an optional package that measures the call-level performance. It is contained in all SD objects, and in some link and VP objects. For the objects of class Link, the package measures the activity for calls that follow the link but not a VP that uses the same link. For VP objects, the package measures the activity of call requests that use the VP. The attributes of the callPerformancePackage are the following: activeCircuits, callArrivalRate (average arrival rate of requests in calls/min), callArrivedCounter (counter of call requests), callResourceBlockedCounter (counter of calls blocked due to resource unavailability), callErrorBlockedCounter (counter of calls blocked due to protocol errors, e.g., time-outs, etc.), callBlockingRate (average rate of calls blocked for any reason in calls/min), setupTime (average time to establish the connection in milliseconds), holdingTime (average duration of connections in seconds), numExchangedMessages (average number of messages that have been exchanged to setup the connections, as an indicator of the processing required for each connection), and measureInterval (the time in which the above averages are computed in seconds). All quantities are measured separately for each traffic class, and then a total over all classes is computed.

The cellPerformancePackage measures cell-level performance. The attributes cellTransmittedCounter, cellTransmissionRate, cellDroppedCounter and cellDroppedRate measure the number of cells transmitted or blocked and their respective time averages. The attribute avgCellDelay measures the average time from the reception till the transmission of cells from the switch. The package is included in objects of class VcEntity, and in this case, only the cells belonging to the VC are measured. As an option, it can also be included in objects of class Link, SourceDestination or VirtualPath. In the latter case, a sum of the attributes over all VC objects that belong to the Link/SourceDestination/VirtualPath is computed, and the respective attributes of the Link/SourceDestination/VirtualPath objects are updated.

The package admissionControllerPackage is mandatory for output link and VP objects. It describes the state of the admission controller, which is located at the output links (for switches with output buffering) and at all VP source points. The package contains the following attributes: networkingCapacity (the schedulable region for link objects or the contract region for VP objects), admissionControllerOperatingPoint (the operating point of the admission controller given the established calls for each traffic class), admissionControlPolicy, admissionControllerOperationalState (enabled (call requests allowed to go through and allocate bandwidth) or disabled) and admissionControllerAdministrativeState.

The class ConnectionMgmt contains attributes that control the operation of the local signalling entity. There is only one instance of this class in every agent. Attributes of this class are the following: signallingProcessingLoad (an index of the call processing load observed by the signalling processor), maxSignallingProcessingLoad (the maximum signalling load value allowed, beyond which the signalling processor denies all call establishment requests), signallingRetransmitTimeout (the time-out value in milliseconds for retransmitting a message if no reply has been received), and signallingCallSetupTimeout 
(the maximum acceptable setup time in milliseconds for a call establishment. If the time to establish a circuit is more than the current value, the circuit is forced to a tear-down). The single instance of this class is also used to contain four other container objects of class LinkMgmt, SourceDestinationMgmt, VirtualPathMgmt, and VirtualCircuitMgmt. There is only one instance from each of these four classes, which is used to contain all objects of class Link, SourceDestination, VirtualPath, and VirtualCircuit, respectively.

As discussed in the previous section, the MIB of every agent resides in the D-plane. Managed Objects use the information stored in the D-plane to update their state. For example, the Managed Objects of class VcEntity represent the Virtual Circuit object that was created in the D-plane by the signalling system. The attributes of the managed object mirror the state of the corresponding real object. In the same manner, the MO of class VirtualPath contains attributes that reflect the state of the corresponding real objects (VP Configuration, Contract Region and Admissible Load Region). An MO of class Link, uses the object Schedulable Region (among other information), to reflect the state of the link Schedulable Region on one of its attributes. Additional processing of events (such as VC creation, etc.) inside the agent can provide the necessary call-level performance related properties (such as call arrival rates). These might not be readily available from other objects of the D-plane (see [ANE94] for more details).

The purpose of the above description was to give an overview of the managed object classes and attributes for performance management. For simplicity, we omitted the definition of associated thresholds for each performance variable that can trigger notifications in case of threshold crossing [ISO92]. Such definitions can be easily incorporated in the above model.

\section{EXPERIMENTAL PLATFORM}

\subsection{The Xunet ATM Testbed}

Xunet is one of the five Gigabit testbeds sponsored by the Corporation for National Research Initiatives. It has been deployed by AT\&T in collaboration with several universities and research laboratories in the continental United States [FRA92]. The topology of the network is shown in Figure 4. The network links are currently rated at $45 \mathrm{Mbps}$ and are gradually substituted with $622 \mathrm{Mbps}$ links. Access at every node is provided by $200 \mathrm{Mbps}$ network interfaces. A variety of standard interfaces (TAXI, HiPPI, etc.) is under development and will be available in the near future. A workstation serves as the switch Control Computer (CC) at each network node. The CC runs the switch control software that performs signalling, control and fault detection functions.

\subsection{The Xunet VP Signalling and Admission Control Architecture}

Xunet supports five traffic classes. Class 1 is used for high priority control messages and is given absolute priority by the link scheduler (multiplexer). Class 2 is used for Video service, Class 3 for Voice, Class 4 for priority data and Class 5 for bulk data [SAR93].

A signalling system very similar in characteristics with the CCSS\#7 (Common Channel Signalling System) has been installed on Xunet. The system allows virtual circuit establishment with best effort resource allocation in a single pass. An admission controller operates on every output link. The necessary Schedulable Region and Admission Control Policy objects are downloaded from a management station. The admission control policy used is complete sharing [HYM93a].

Virtual Path establishment is also done in one pass with best effort resource allocation. When the VP has been established, an admission controller is activated at the source node of the VP that uses the allocated contract region for admission control decisions. The admission control policy is again complete sharing. A signalling channel is also established between the two VP termination points to carry call establishment requests over the VP. It operates in the same way as the signalling channel used on every physical link. As a result, from the point of view of the signalling system, VPs are considered as regular links with only minor differences. 
Every Contract Region can be changed dynamically. The deallocation or allocation of additional resources is performed in the same way as in the VP establishment phase. Finally, when a VP is removed, the Contract Region is returned to the Schedulable Regions of the links along the path, all VCs using the VP are forced to termination and the VP signalling channel is destroyed.

\subsection{The Xunet OSI Management System}

From the five functional areas covered by the OSI management model, we have chosen to implement a configuration, fault and performance management architecture for Xunet (the remaining functional areas being security and accounting management). The configuration and fault management architecture enables us to monitor closely all the network switches for hardware faults, such as link level errors, buffer overflows, etc. The performance management architecture builds on the performance management model (managed object definitions and behavior) that was presented in the previous section.

As the basis of our OSI Management system, we have selected the OSIMIS software [KNI91]. Our implementation expanded the agent with managed objects for Xunet and the management applications to include powerful graphics that depict the operational state of the network and a control interface that facilitates the overall management task. The management applications run at Columbia University. TCP/ IP sockets are used at the transport layer to connect to the agent at each site. Inside the agents, communication between logical and physical objects is achieved by using a proprietary protocol between the agent and the Xunet switch. For this purpose, we use UDP/IP packets over a local Ethernet.The structure of the system is shown in Figure 3.

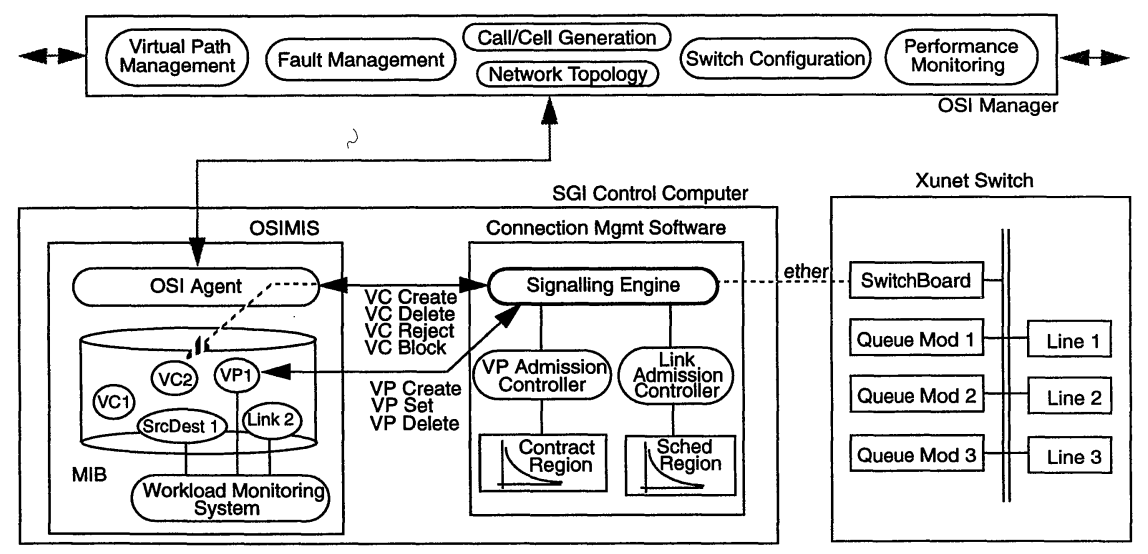

Figure 3: Structure of the Xunet Management System.

\subsection{The OSI Agent}

The OSI agent contains managed objects for configuration, fault and performance management. The agent consists logically of two major groups of Managed Objects.

\subsubsection{Hardware Configuration and Fault Management Group (HCFMG)}

For the purpose of configuration and fault management, we have implemented managed object classes for each Xunet hardware module, such as SwitchBoard, QueueModule, etc. Each module is polled at regular time intervals by the agent to detect possible faults. A hardware fault triggers a notification inside the agent, which in turn can generate a CMIS Event Report primitive if the appropriate Event Forwarding Discriminator object has been created by the manager [ISO91]. Currently, more than 300 different hardware 
errors can produce an equal number of event reports. This wealth of information provides the manager with extensive fault monitoring capabilities. The configuration and state of the hardware modules is obtained from the Xunet switch every 20 seconds. The information is processed internally to update the corresponding managed objects.

The set of the hardware managed objects also gives complete configuration information of every switch. The management applications can display graphically the configuration and indicate the location of every generated event report.

\subsubsection{Performance Management Group (PMG)}

The PMG consists of a set of managed objects that monitor closely the performance of Xunet both at the cell and at the call level. All call level information is obtained from the local signalling entity. The OSI agent receives four types of events: VC-Create, VC-Delete, VC-Blocking (no network resources) and VC-Rejection (any other cause), with the appropriate parameters. Upon a VC creation event, a Managed Object of class VcEntity is created inside the MIB that contains all the available information on this VC. The object is related to the appropriate Link, SourceDestination or VirtualPath objects. Every 30 seconds, the Xunet switch is scanned to compute the number of cells transmitted or dropped for each VC. At the same time we use this information to update the total number of cells transmitted or lost on a link, SD pair or VP based on the relations defined when the VC was created. The VC object is removed by a deletion event.

All 4 event types cause the agent to update some internal counters in the corresponding Link/SD/VP objects. Additional processing is performed at a regular time interval (controllable by the manager through the measureInterval attribute, and usually set to 30 seconds). At that time, the agent calculates the values for the performance related attributes, taking into account only the events that occurred during the past interval. For example, when a VC is created, a counter that registers the call arrivals is incremented. At the end of the 30 second period, the arrival rate is calculated, and the counter is reset to zero. All other attributes are calculated in a similar fashion.

VP management functions originate at the network manager site. When the management application issues an M-Create command with the appropriate parameters, a VP managed object inside the MIB is instantiated, and the Xunet signalling entity is informed to initiate a VP setup procedure. VPs can be subsequently modified by the M-Set command operating on the appropriate object, and deleted with an M-Delete command.

Parameters of the signalling entity are controlled through M-Set operations on attributes of the ConnectionMgmt object. Each Set operation causes a control message to be sent from the agent to the signalling entity.

\subsection{The OSI Manager}

Xunet is currently monitored and controlled through a Motif/X-Toolkit-based application. The same application is used to run controlled call and cell generation experiments on Xunet. It consists of six tightly interconnected subsystems (Figure 3). Every subsystem contains the appropriate display tools and management interface functions for a specific management task:

1. Switch Configuration: Displays the hardware configuration of the switch using information from the objects of the HCFMG.

2. Fault Management: Receives OSI Event reports from the agents, that are related to hardware problems, and uses the Switch Configuration subsystem's display functions to inform the manager about the nature and location of the problem.

3. Network Topology: Displays a map of the network, with all switches, links and attached user-network interfaces. The displayed objects can be selected and observed independently. Support is also provided for displaying the route and configuration information of VPs. 
4. Virtual Path Management: The manager is able to create and subsequently control VPs with MCreate and M-Set operations. The VP control task is guided by the observations obtained from the Performance Monitoring system.

5. Performance Monitoring: Collects the information that is provided by the PMG objects in each node and displays it using the functions of the Network Topology subsystem. The information can be either displayed in textual form, or graphically. In the latter case, we use time series plots that are updated in real-time. The plots allow us to observe the performance "history" of the network and the effects of VP management controls.

6. Call and Cell Generation: The Xunet signalling entities contain a call generation facility. A managed object inside the local agent makes it possible to control the call generation parameters in terms of destination nodes, call arrival rate and call holding time on a per traffic class basis. The call generation system can also be linked to the Xunet cell generator for real-time cell generation.
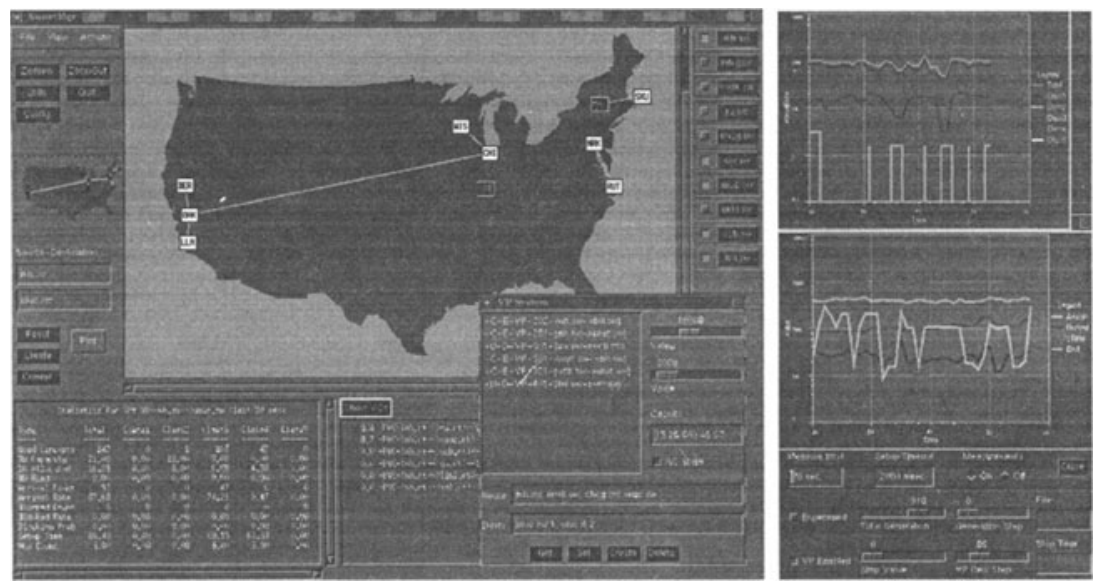

Figure 4: The Xunet Management Console displaying the call level performance.

\section{PERFORMANCE}

We are currently using the management system to run controlled experiments on Xunet to study the call level performance of the network, such as the performance of the signalling system and the network throughput under various VP distribution policies. Call level experiments consist of loading the signalling system with an artificial call load. A Call Generator on every switch produces call requests with exponentially distributed interarrival and holding times. In the remaining of this section we will focus on the objective of performance management at the call level and will demonstrate results from various calllevel experiments conducted on Xunet.

\subsection{Semantics of Performance Management}

The objective of performance management at the call level can be summarized in the following:

- Minimize call blocking due to unavailability of network resources. This unavailability can be caused by several factors including a faulty link, a poor VP distribution policy, a routing malfunction, an overloaded signalling processor, etc. 
- Minimize the call setup time. The call setup time is perceived by the user as a measure of the quality of service offered by the network. High call set up times may prompt the user to hang-up leading to loss of revenue for the service provider.

Increasing the bandwidth of a VP results in reducing the signalling load on the network, but also in a possibly reduced network throughput. Our main goal is to evaluate this fundamental trade-off between network throughput and signalling load and choose a VP distribution policy that results in the best overall performance.

The manager collects measurements in regular time intervals, and evaluates network performance, either in a per SD-pair basis or by looking at individual nodes, links or VPs. If the performance is not satisfactory (high blocking, high call setup times and high signalling load), the manager can apply the following controls:

1. Create a VP between two nodes and allocate resources to it. This action alleviates the intermediate nodes from processing call requests and decreases the call setup time.

2. Delete a VP responsible for the non-satisfactory performance. This course of action may be taken because the maximum number of VP terminations has been reached and new VPs cannot be created in the system, or because there is no offered load to the VP, or because a new VP distribution policy has been decided and the VP topology must change.

3. Change the allocated networking capacity of a VP either by releasing a part of or increasing the allocated resources. This control is performed when the load offered to the VP has been reduced or increased.

4. Change signalling parameters, such as the time-out for call setups, the time-out for message retransmissions and the maximum allowed signalling load (which is a window-type control on the number of requests handled by the signalling processor). These parameters affect the call blocking rates, but also the average call setup time.

With the above in mind, the call-level experiments have been separated in two major phases. In the first phase, we measure the performance of the signalling system without using VPs. This experiment allows us to find suitable values for the parameters of the signalling entities that give the highest call throughput. The second phase builds upon the first phase, and attempts to determine the call throughput by measuring the performance of the network with VPs in place.

\subsection{Performance of the Signalling System for Virtual Circuit Set-Up}

In this experiment, the network was loaded with an artificial call pattern. Our goal was to measure the performance of the signalling system under heavy call arrivals. For each call, the signalling system sets up a VC by traversing all the nodes in the path and patching the appropriate connections in each node. Call generation is controllable for each destination in terms of the call arrival rate and call holding time for each of the five traffic classes supported by Xunet. The network was homogeneously loaded from five sites (Murray Hill, Rutgers U., U. of Illinois, U.C. Berkeley and Livermore) with Poisson call arrivals and an exponential holding time with a mean of 3 minutes. We used only video calls (assumed to consume a peak of $6 \mathrm{Mbps} / \mathrm{call}$ ) and voice calls (64 Kbps/call) in a ratio of 1:10 (i.e., the arrival rate of voice calls is 10 times greater). All the links in the experiment described here are of $45 \mathrm{Mbps}$ capacity. The schedulable region (SR) of each link assumed to be given by a two dimensional hyperplane. We used peak rate allocation, and according to this scheme, the SR can accommodate a maximum of 7 video calls or 703 voice calls. The admission control policy used was complete sharing [HYM93a].

Figure 5 shows the measurements obtained by gradually increasing the call generation rate. Each measurement (throughput, call setup time, call blocking etc.) is computed by adding (or averaging) the respective measurements for the video and voice traffic. Both the call throughput and call blocking due to resource unavailability (the "Throughput" and "Blocked Percentage" curves) rise almost linearly with the call arrival rate. The sudden drop in the total call throughput is due to the overloading of the signalling system with call setup requests. At that point, the call setup time and the percentage of calls blocked due 

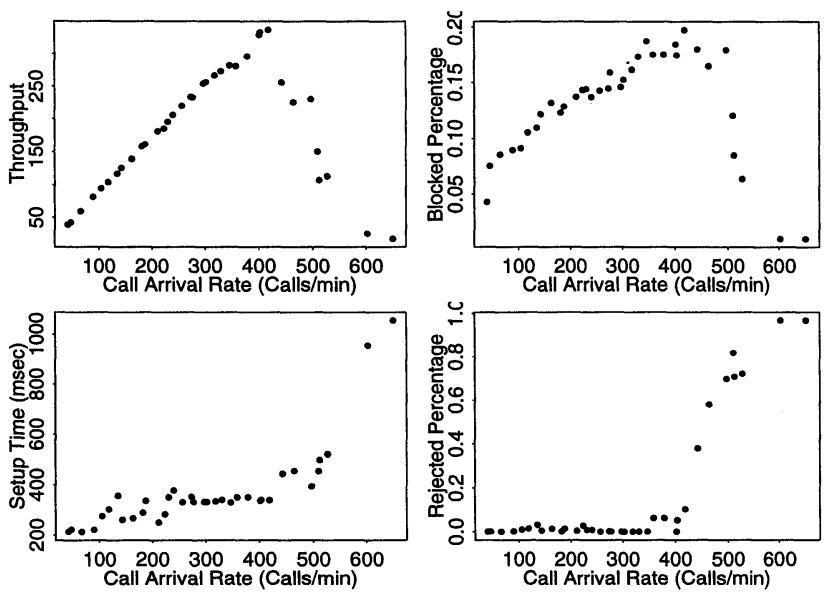

Figure 5: Performance of the Signalling System.

to congestion of the signalling system (the "Rejected Percentage" plot) start to rise sharply. The "BlockedPercentage" curve drops because the strain has now been moved from network transport to call setup, and thus, calls contend for signalling resources rather than networking resources. During overload, only a small percentage of the total call attempts is actually established, and therefore, the probability that these calls will find no networking capacity available is diminished. In the extreme situation, all calls are blocked while the networking capacity of all links is unused.

The congestion situations seem to appear first at the Newark and Oakland switches, that are the first to become overloaded with call request messages. It is therefore essential for the network manager to regulate the call arrival rate at the entry points in the network. This can be done by setting an appropriate value for the maxSignallingProcessingLoad attribute of the ConnectionMgmt object. The signalling load is computed from the number of signalling messages received and transmitted from the switch in the unit of time. If the load reaches the maxSignallingProcessingLoad value, a fraction of the incoming call requests are discarded. We have found experimentally that by restricting the signalling load to about 450 messages per minute at the nodes connected to the call generators, the network operates within the capacity of the signalling processors.

\subsection{Performance Trade-off due to VP Capacity Allocation}

This experiment had the objective of studying the trade-off between the network throughput and the allocation of networking capacity to VPs. It was performed on the east coast segment of the network. This four node segment consists of two nodes in MurrayHill (called MHEX and MH), one node in Newark (NWRK), and one at Rutgers University (RUTG) connected in tandem.

The generation ratio between Class 2 and Class 3 calls was 1:100. The call arrival rate from each call generator was kept fixed throughout the experiment. The generator at MHEX produces traffic to NWRK (180 calls/min) and RUTG (210 calls/min). The generator at MH produces only traffic to RUTG at 180 calls/ min. The generator at RUTG produces traffic to NWRK at $180 \mathrm{calls} / \mathrm{min}$. One VP is established from MHEX to RUTG (Figure 6).

Only the traffic from MHEX to RUTG is allowed to follow the VP. Calls that find the VP full and the calls from other traffic generators follow a hop by hop call setup procedure, that is, trying to secure bandwidth on each hop. By contrast, calls that follow the VP contest for bandwidth only at the VP source node. The 


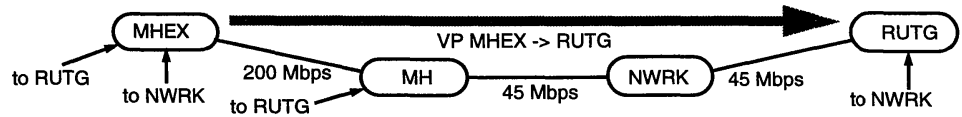

Figure 6: Network Topology for the VP experiment.

capacity of the VP varies from 0 to 100 percent of the capacity of the smallest link on the VP (the MHNWRK link which is rated at $45 \mathrm{Mbps}$ ). When the VP capacity is at $100 \%$ only calls from MHEX to RUTG are allowed, since all other calls find no available resources to proceed to their destination. When the VP capacity is reduced to 0 , all calls are attempting the regular VC setup procedure. Figure 7 shows the obtained measurements. The throughput curve reveals that the maximum throughput is attained when
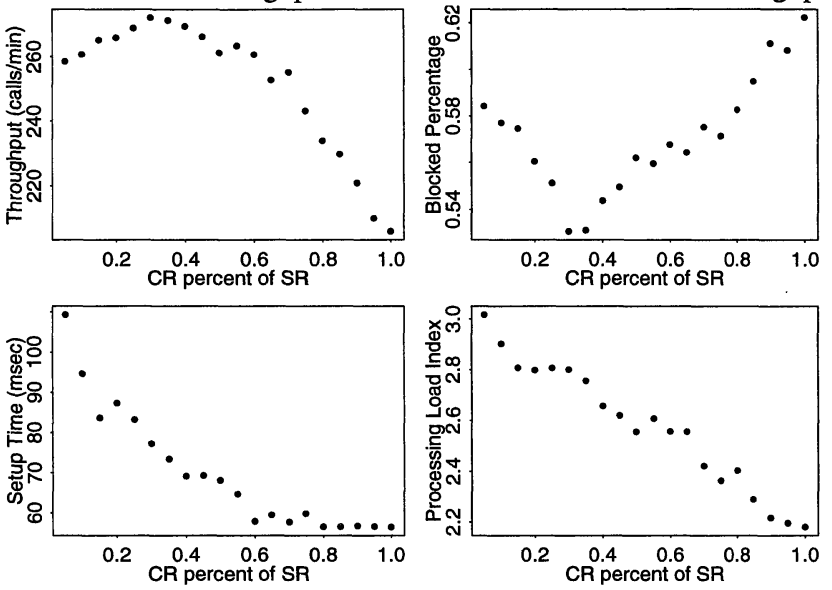

Figure 7: Virtual Path performance vs. allocated networking capacity.

the VP contract region is approximately 30 percent of the link schedulable region. This happens because below that value, an increasing amount of call requests from MHEX to RUTG find the VP full and use the regular VC setup procedure, thereby forcing the signalling entity at MH and NWRK into an overload state, that causes high call setup times and higher blocking. When the VP contract region increases above 30 percent, the throughput drops slowly as the extra capacity allocated for the VP is partially unused, and as a result a larger percentage of the interfering traffic (that does not follow the VP) is blocked. The fourth plot depicts the average number of signalling messages needed to establish (or reject) an incoming call. The numbers drop as the VP increases in capacity, as calls from MHEX to RUTG follow the VP and use less hops to reach the destination.

\section{SUMMARY AND FUTURE WORK}

A basic model for the performance management of VP connection services for ATM broadband networks was presented. A set of managed object classes following the OSI standard for network management with complete attribute structure was proposed. The call-level model enables the network manager to retrieve information from agents installed in the network, make decisions based on the configuration and performance observations, and apply a set of controls if the observed performance can be improved. These controls include setting the operating parameters of the signalling code and changing entirely or in part the distribution of the VPs in the system.

Our model was fully implemented on the Xunet ATM testbed. The manager is able to observe the call level 
performance of Xunet from a dedicated management tool. We have presented some aspects on the call level performance of Xunet and demonstrated the behavior of the network when VPs are in use.

We are currently working on an algorithm for an automated tool that observes the offered call load and the call-level performance related properties and makes decisions regarding the VP distribution policy and the operating parameters of the signalling software. Such a system will significantly facilitate the performance management task for a network with a large number of nodes and VPs.

This work was funded in part by NSF Grant CDA-90-24735, and in part by a Grant from the AT\&T Foundation.

\section{REFERENCES}

[ANE94] Nikos G. Aneroussis and Aurel A. Lazar, "Managing Virtual Paths on Xunet III: Architecture, Experimental Platform and Performance", CTR Technical Report \#369-94-16, Center for Telecommunications Research, Columbia University, 1994, URL: "ftp://ftp.ctr.columbia.edu/CTR-Research/comet/public/papers/94/ANE94.ps.gz".

[ANE93] Nikos G. Aneroussis, Charles R. Kalmanek and Van E. Kelly, "Implementing OSI Management Facilities on the Xunet ATM Platform," Proceedings of the Fourth IFIP/IEEE International Workshop on Distributed Systems: Operations and Management, Long Branch, New Jersey, October 1993.

[FRA92] A.G. Fraser, C.R. Kalmanek, A.E. Kaplan, W.T. Marshall and R.C. Restrick, "Xunet 2: A Nationwide Testbed in High-Speed Networking," Proceedings of the IEEE INFOCOM'92, Florence, Italy, May 1992.

[HYM91] Jay M. Hyman, Aurel A. Lazar, and Giovanni Pacifici, "Real-time scheduling with quality of service constraints," IEEE Journal on Selected Areas in Communications, vol. 9, pp. 1052-1063, September 1991.

[HYM93a] Jay M. Hyman, Aurel A. Lazar, and Giovanni Pacifici, "A separation principle between scheduling and admission control for broadband switching," IEEE Journal on Selected Areas in Communications, vol.11, pp. 605-616, May 1993.

[HYM93b] Jay M. Hyman, Aurel A. Lazar, and Giovanni Pacifici, "Modelling VC, VP and VNBandwidth Assignment Strategies in Broadband Networks", Proceedings of the Workshop on Network and Operating Systems Support for Digital Audio and Video, Lancaster, United Kingdom, November 3-5, 1993, pp. 99-110.

[ICM93] ICM Consortium, "Revised TMN Architecture, Functions and Case Studies", ICM Deliverable 5, 30 September 1993.

[IET94] Internet Engineering Task Force, “Definition of Managed Objects for ATM Management”, Internet Draft Version 7.0, March 9, 1994.

[ISO91] Information Processing Systems - Open Systems Interconnection, "Systems Management - Fault Management - Part 5: Event Report Management Function,” July 1991. International Standard 10164-5.

[ISO92] Information Processing Systems - Open Systems Interconnection, "Systems Management - Performance Management - Part 11: Workload Monitoring Function”, April 1992. International Standard 10164-11.

[LAZ92] Lazar, A.A., "A Real-Time Management, Control and Information Transport Architecture for Broadband Networks", in Proceedings of the 1992 International Zurich Seminar on Digital Communications, Zurich, Switzerland, March 1992.

[LAZ93] Lazar, A.A. and Stadler, R.,"'On Reducing the Complexity of Management and Control in Future Broadband Networks", Proceedings of the Fourth IFIP/IEEE International Workshop on Distributed Systems: Operations and Management, Long Branch, New Jersey, October 1993.

[KNI91] George Pavlou, Graham Knight and Simon Walton, "Experience of Implementing OSI Management Facilities," Integrated Network Management, II (I. Krishnan and W. Zimmer, editors), pp. 259-270, North Holland, 1991.

[OHT93] Ohta, S., and Fujii, N., "Applying OSI System Management Standards to Virtual Path Testing in ATM Networks", Proceedings of the IFIP TC6/WG6.6 Third International Symposium on Integrated Network Management, San Francisco, California, 18-23 April, 1993.

[SAR93] H. Saran, S. Keshav, C.R. Kalmanek and S.P. Morgan, "A Scheduling Discipline and Admission Control Policy for Xunet 2", Proceedings of the Workshop on Network and Operating Systems Support for Digital Audio and Video, Lancaster, United Kingdom, November 3-5, 1993.

[TSU92] Tsuchida, M., Lazar, A.A., Aneroussis, N.G., "Structural Representation of Management and Control Information in Broadband Networks", Proceedings of the 1992 IEEE International Conference on Communications, Chicago IL., June 1992. 
Nikos G. Aneroussis was born in Athens, Greece in 1967. He received the Diploma in Electrical Engineering from the National Technical University of Athens, Greece, in May 1990, and the M.S. and M.Phil. degrees in Electrical Engineering from Columbia University, New York, NY in 1991 and 1994. Since 1990, he is a graduate research assistant in the department of Electrical Engineering and the Center for Telecommunications Research at Columbia University, where he is currently pursuing the Ph.D. degree. His main research interests are in the field of computer and communication networks with emphasis on management architectures for broadband networks and network performance optimization. He is a student member of the IEEE and a member of the Technical Chamber of Greece.

Aurel A. Lazar is a Professor of Electrical Engineering and the Director of the Multimedia Networking Laboratory of the Center for Telecommunications Research, at Columbia University in New York.

Along with his longstanding interest in network control and management, he is leading investigations into multimedia networking architectures that support interoperable exchange mechanisms for interactive and on demand multimedia applications with quality of service requirements.

A Fellow of IEEE, Professor Lazar is an editor of the ACM Multimedia Systems, past area editor for Network Management and Control of the IEEE Transactions on Communications, member of the editorial board of Telecommunication Systems and editor of the Springer monograph series on Telecommunication Networks and Computer Systems. His home page address is http://www.ctr.columbia.edu/ aurel. 